\title{
Development of Front-end Software for Beam Parameters Measurement for Indus-2 Electron Synchrotron
}

\author{
S. Yadav \\ Raja Ramanna Centre for \\ Advanced Technology, Indore, \\ India \\ \& \\ Homi Bhabha National Institute, \\ Mumbai, India
}

\author{
T.A. Puntambekar \\ Raja Ramanna Centre for \\ Advanced Technology, Indore, \\ India
}

\author{
P.V. Varde \\ Bhabha Atomic Research Centre, \\ Mumbai, India
}

\begin{abstract}
An electron synchrotron with high brilliance requires monitoring of the electron beam parameters continuously. The state of the machine may be identified by measuring the beam parameters like betatron tune, synchrotron tune, beam orbit, instabilities level etc. To measure the machine status for Indus-2, a synchrotron radiation source at RRCAT, Indore, India, an integrated measurement scheme has been developed. This work involves the design of six different measurement modules, interfacing the hardware to initialize the measurement and acquisition of the data from hardware into software. The software acquires the beam position and beam intensity data in time and frequency domains. Signal processing techniques like point invariant concatenation of acquired data, low pass filtering, curve fitting, multi-peak detection etc. are applied on the acquired data to measure the beam parameters simultaneously. With the development of this software, more than 800 parameters are monitored online and logged.
\end{abstract}

\section{Keywords}

Betatron tune, synchrotron tune, beam lifetime, coupled bunch instability etc.

\section{INTRODUCTION}

The electron synchrotron is becoming an important tool as a synchrotron radiation source (SRS) in various research areas. Current trend in SRS is to go for high beam current along with a decrease in beam size. Indus- 2 is an electron synchrotron radiation source of nominal electron energy of $2.5 \mathrm{GeV}$ and a critical wavelength of about 1.986 Angstroms. A booster synchrotron is used as an injector with $550 \mathrm{MeV}$ beam energy for injection into Indus 2. After the accumulation of beam current in Indus-2, beam energy is ramped up to $2.5 \mathrm{GeV}$ [1]. The machine parameters of Indus-2 are given in the table 1 [2] [3]. It is imperative in any SRS to store the electron beam current with minimum beam loss. During the day to day operation of Indus-2 storage ring, difficulty was being faced sometimes in accumulating higher beam current and in beam energy ramping. After examining, it was found that the beam parameters are deviating from their desired specification during operation. For smooth beam operation, maintaining the beam parameters within a desired range plays an important role in avoiding the beam loss.
Table 1. Summary of Indus-2 beam parameters

\begin{tabular}{|c|c|}
\hline Parameter & Specification \\
\hline Beam energy & $0.550-2.5 \mathrm{GeV}$ \\
\hline Revolution frequency & $1.7382 \mathrm{MHz}$ \\
\hline Beam current & $200 \mathrm{~mA}$ \\
\hline Betatron tune & $9.29,6.14$ \\
\hline Harmonic Number & 291 \\
\hline RF frequency & $505.8 \mathrm{MHz}$ \\
\hline Damping times @ & $4.74 \mathrm{~ms}$ (horizontal plane), \\
$2.5 \mathrm{GeV}$ & $2.62 \mathrm{~ms}$ (vertical plane), \\
& $2.28 \mathrm{~ms}$ (longitudinal plane) \\
\hline
\end{tabular}

At high beam current accumulation, the impedances of the machine may be resonant with the beam frequencies (in the beam spectrum) leading to coupled bunch beam instability in longitudinal and transverse planes. In an accelerator, at high beam currents, transverse instabilities of the beam adversely affect the machine performance. The levels of these instabilities are dependent on the various machine parameters. In Indus-2, 291 modes are possible in which bunches interact with each other and cause instability. To get the information about the machine state during the beam operation, knowledge of beam parameters with all modes of instabilities is necessary. This paper describes the development of the beam diagnostics front-end software for measurement of above stated parameters simultaneously. The system also provides the data logging of beam parameters. These data logging and retrieval systems enable accelerator physicists to easily access the logged data for studies of the machine optics.

\section{METHODOLOGY}

The integration of the beam diagnostics subsystem to developed integrated front end is a challenging task in view of the requirement of accuracy and speed of measurement. In the integrated measurement scheme, a time gape is required between coupled bunch instability measurement and tune measurement. It is because, beam excitation is required to measure the betatron tune and synchrotron tune, whereas the natural beam excitation level is measured for the measurement of coupled bunch instabilities. Hence the beam oscillation due to excitation needs to damp out to desired level before measurement of coupled bunch instabilities. 
To analyze decay of beam oscillation a Simulink model has been developed. The mathematical equation for beam motion may be given by equation of simple harmonic motion as follows [4],

$$
\frac{\mathrm{d}^{2} \mathrm{x}}{\mathrm{dt}^{2}}+2 D \frac{d x}{d t}+\omega^{2} x=0
$$

Where, $\mathrm{x}$ is beam displacement, $\mathrm{D}$ is the damping term and $\omega$ is frequency of oscillation. In the case of the beam excitation due to wake field, the equation may be rewritten as [4],

$$
\frac{\mathrm{d}^{2} \mathrm{x}}{\mathrm{dt}^{2}}+2(D-G) \frac{d x}{d t}+\omega^{2} x=0
$$

Where, $\mathrm{G}$ is the excitation term due to wake field.

The Simulink model of the beam oscillation is shown in fig: 1 .

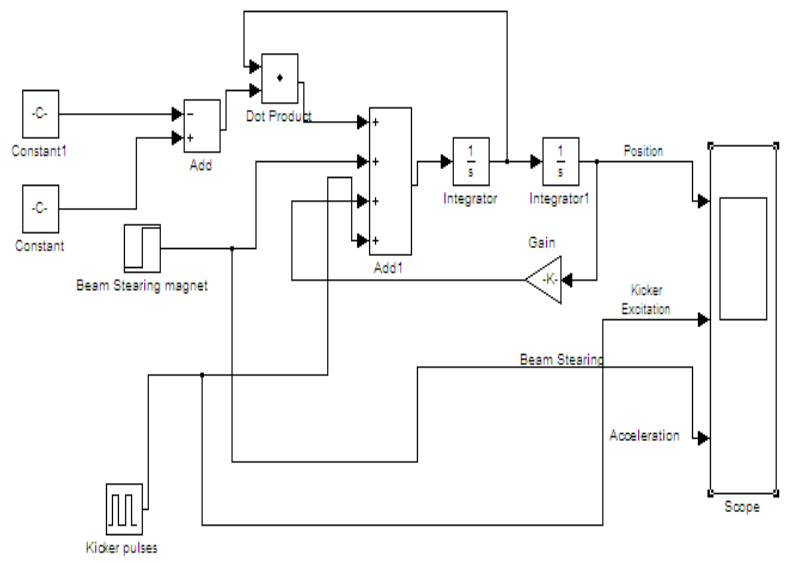

Fig. 1: Simulink model for analysis of beam motion

The parameters of the Indus- 2 beam have been used in Simulink model to find out time duration for damping of beam oscillation below noise level [2] [3]. After analysis of beam oscillation due to kicker pulse excitation, it is observed that beam oscillation is decay down to noise level within 0.5 Sec. Hence a time gap of $1 \mathrm{Sec}$ is used between coupled bunch instability measurement and tune measurement considering the dynamic behavior of accelerator system. Due to less coupling between oscillation of transverse plane (betatron oscillation) and oscillation of longitudinal plane (synchrotron oscillation), measurement of betatron tune and longitudinal coupled bunch instability measurement may be done in sequence. Similar is true for synchrotron tune measurement and measurement of transverse coupled bunch instabilities.

The separate modules of beam parameter measurement for the integrated measurement system have been developed. These modules used for beam parameters measurement in a sequence based on the above analysis. The modules of measurement system are as follows,

\subsection{Beam Current and Beam Energy Acquisition Module}

This module acquires the beam current and beam energy data from the server using the TCP IP network. The beam energy and beam current data are converted in numerical value and used for deciding the excitation power of the tune measurement system and in the calculation of beam lifetime.

\subsection{Beam Life Time Measurement Module}

The beam lifetime measurement module utilizes the acquired beam data and fits an exponential curve to the beam current array. The beam lifetime is calculated by this module so that the uncertainty in the beam lifetime measurement is within $2 \%$. The number of beam current samples used for the beam lifetime calculation depends on the given uncertainty and noise in beam current data [5].

\subsection{Betatron Tune Measurement Module}

This is an important parameter for the machine. The change in the tune point from its optimum value may result in the decrease in the beam lifetime or beam loss. The transverse optics of the accelerator governs the tune point of machine. The measurement module is an active system. It energizes the kicker to kick the beam and measures the transverse oscillation for tune measurement. The level of excitation for kicker is adjusted by the module based on the beam energy data.

\subsection{Synchrotron Tune Measurement Module}

Like the betatron tune, synchrotron tune depends on the longitudinal dynamics of the machine. This module excites the electron beam in longitudinal direction and measures the longitudinal oscillation.

\subsection{Transverse Instability Measurement Module}

This module acquires the spectrum of beam position data in horizontal and vertical plane. The acquired data is partitioned into 145 parts and analyzed to extract the amplitude of transverse beam excitation. The 291 modes of excitation are measured in each plane. The average instability level and maximum instability level have been calculated in this module.

\subsection{Longitudinal Instability Measurement Module}

Like the transverse instability module, this module acquires the spectrum of intensity signal and identifies the amplitude of the longitudinal excitation for 291 modes.

\section{SYSTEM DESCRIPTION}

The schematic diagram of the beam parameters measurement system is shown in fig.2. The measurement system employs a spectrum analyzer equipped with a tracking generator for beam excitation signal for tune measurement. The tracking generator RF output is then fed to an RF processing front end for produce four output kick signals which are amplified and drive the strip line kicker. Another strip-line located in long straight section (LS-2) of the Indus-2 ring is used as a beam position monitor to observe the betatron oscillation and synchrotron oscillation [6]. These signals are analyzed by a spectrum analyzer. For observation of coupled bunch modes, beam position and intensity signal are also fed to a real time spectrum analyzer for measurement of coupled bunch instabilities in longitudinal and transverse plane. 


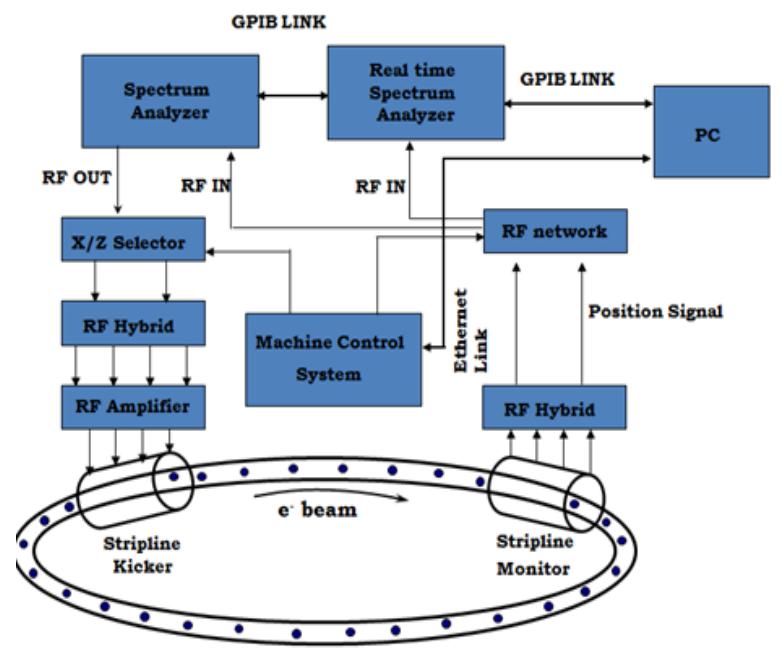

Fig. 2: Schematic diagram of beam parameters measurement system

The beam current, beam energy and beam orbit data are acquire from the server using the TCP IP network. These data are used to calculate the beam lifetime, RMS beam orbit and maximum close orbit distortion. The measurement results are displayed online and logged in MS excel sheet.

\section{RESULT AND ANALYSIS}

The integrated diagnostics software has been developed and deployed to measure beam parameters simultaneously. The measurement speed is < $28 \mathrm{Sec}$ per measurement. The resolution of betatron and synchrotron tune measurement is 1 part per thousand. The RMS uncertainty of coupled bunch mode amplitude is $<5 \mathrm{~dB}$. The beam lifetime measurement accuracy is $2 \%$. RMS closed orbit distortion (COD) is measured with a resolution of $<15$ micrometer.

This system is being regularly used to get the information about beam operation stability and repeatability. The system has played an important role in beam loss control during beam energy ramping. Typical graphs of measurements are shown in Fig. 3.

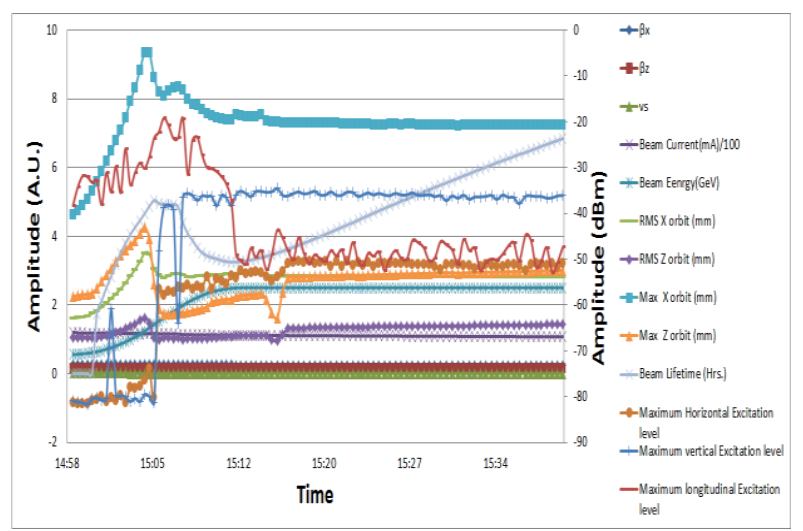

Fig. 3: Typical measurement result during beam operation
During the database collection and analysis it was found that the beam parameters betatron tune and COD affects beam operation significantly. It was also observed that coupled bunch mode number 229 in the horizontal plane was excited to more than $30 \mathrm{dBm}$ from nominal level during high current operation in Indus-2. Partial beam loss is observed in the presence of this instability, which is controlled by changing the sextupole magnet current.

\section{CONCLUSION}

The integrated user interface of measurement system has been developed to acquire the data of the system. A database is created from the system in various machine operating conditions. Logged beam parameters analysis will help in the development of a user support system to optimize the machine and improve the reliability.

\section{ACKNOWLEDGMENTS}

The authors thank Mr. C. P. Navathe and Dr. P. D. Gupta for their encouragement and support to complete this work. They also thank colleagues of Beam Diagnostics Section of RRCAT for their help during development and beam experiments conducted in the Indus- 2 storage ring. The authors also thank colleagues of Accelerator Controls Section of RRCAT for their support for beam parameter interface with developed software. Authors also wish to thank shift crew members of the Indus accelerator complex for their support during beam experiments.

\section{REFERENCES}

[1] G. Singh, G. K. Sahoo, D. Angel, B. Singh, A. D. Ghodke and P. Kant 1997 "Synchrotron Radiation Source Indus-2” IJAP 35, 1 183-192

[2] Saroj Jena, S. Yadav, R. K. Agrawal, A.D. Ghodke, Pravin Fatnani, T.A. Puntambekar, "Stabilization of betatron tune in Indus-2 storage ring", Chinese Physics C, arXiv:1307.4512 [physics.acc-ph] (2013)

[3] "Accelerator Activity", http://www.rrcat.gov.in/ technology/accel/indus/indus2.html.

[4] Marco Lonza, "Multi- bunch Feedback Systems", Cern Accelerator School - "Beam Diagnostics" - Dourdan, FRANCE , 28 May - 6 June 2008

[5] X. Huang and J. Corbett, "Measurement of Beam Lifetime and Application for SPEAR3", Nucl. Instrum. Meth. Phys. Res. A 629, 31 (2011) doi: 10.1016/j.nima.2010.10.147

[6] T. A. Puntambekar et al., "Experience of beam diagnostic systems in commissioning stage of Indus-2", Asian Particle Accelerator Conference - 2007 (APAC07) , RRCAT, Indore , Jan. 29 - Feb. 2, 2007, p. 413-415

\section{AUTHOR PROFILE}

S. Yadav has completed his B.E. in electronics in 2003. Currently he is working with the Raja Ramanna Centre for Advanced Technology, Indore, India. He is pursuing the $\mathrm{Ph}$ D. from Homi Bhabha National Institute, Mumbai. 